\title{
COLD NEUTRON INTERFEROMETRY USING MULTILAYER NEUTRON MIRRORS
}

\author{
T. KAWAI
}

Research Reactor Institute, Kyoto University, Kumatori-cho, Osaka 590-0494, Japan

The object of this report is to review the recent results and some perspectives on cold neutron interferometry using multilayer neutron mirrors developed in KUR. The purposes of our research are to develop new experimental techniques, new devices for controlling neutron spin states for opening up a new world of physics. A multilayer neutron polarizer working under a very low external magnetic field (low field magnetic mirror), Funahashi's interferometer, a cold neutron spin echo instrument, a multilayer neutron spin splitter and a cold neutron pulser were developed. The equivalency of the quantum precession induced by the effective gap layer of the multilayer neutron spin splitter and Larmor precession of neutron spin in a magnetic field is established. This idea allows us to develop a cold neutron phase-spin-echo interferometer (a neutron spin interferometer). The neutron spin interferometer and a cold neutron pulser enabled us to carry out the delayed choice experiment for answering the question "how the neutron wave propagates after being split by the semitransparent mirror (wave splitter)". These matters are reviewed in this paper.

PACS numbers: 03.75.-b

\section{Introduction}

A neutron is a fermion with a spin angular momentum of quantum number $1 / 2$ and a magnetic moment, and thus interferes only with itself. The de Broglie wave property of the massive neutron could be extracted successfully in combination of cold neutrons with multilayer neutron mirrors. The ferromagnetic mirrors allow us to control the neutron spin state by their magnetic potential and make various kinds of neutron optical devices for controlling spin states. An artificial multilayer neutron mirror enables us to make a composite mirror which consists of layers of different materials and thicknesses [1]. Multilayer magnetic mirrors working under a very low external field around zero gauss (low field magnetic mirror) are especially useful for cold neutron interferometry [2-4]. The motivation of developing a low field magnetic mirror was the necessity of the magnetic mirror with low electricity which could be used in liquid helium to polarize ultracold neutrons. A low field magnetic mirror placed in the pulsed magnetic field is called a 
multilayer cold neutron pulser or in abbreviation a cold neutron pulser $[5,6]$. This device produces pulsed cold neutrons and works as a non-mechanical chopper.

A Jamin-type neutron interferometer consisting of a pair of two composite. mirrors has been developed by Funahashi et al. [7]. The idea of the micro-neutron interferometer [8] was a helpful hint for this new interferometer. This interferometer in combination with low field magnetic mirrors opens up the road to development of various kinds of cold neutron interferometry [9].

On the one hand the cold neutron spin echo instrument controlled by low magnetic field was also developed from a viewpoint of cold neutron interferometry to investigate the Larmor time during which neutron tunnels through the magnetic thin film [10]. On the other hand, the valid interpretation for neutron state polarized in the horizontal $x-y$ plane was established experimentally [11-15]. The neutron state polarized in $x-y$ plane is expressed as a superposition of the two eigenstates quantized along the vertical direction $z$ (quantization axis). Precession of neutron spin in the $x-y$ plane causes a relative phase shift between two eigenstates. Such a precession of neutron spin is cailed "quantum precession" [18]. From this thought, the multilayer spin splitter was born. It consists of a multilayer magnetic mirror, a gap layer, and a multilayer nonmagnetic mirror. The outer magnetic mirror reflects one spin eigenstate while the inner nonmagnetic mirror reflects the other eigenstate. The gap layer produces a path length difference between two eigenstates, that is, a relative phase shift between them. The relative phase shift corresponds to a quantum precession of the neutron spin.

The equivalency of the quantum precession with Larmor precession in a magnetic field is demonstrated [18]. Using the quantum precession phenomenon, the phase-spin-echo neutron interferometer is developed [18].

In 1978 Wheeler presented the delayed choice thought-experiment to verify Bohr's view concerning the propagating behavior of the photon (boson) after being split through a semi-reflecting mirror (wave splitter) [19]. In that experiment, it is required to establish the condition of whether the second semi-reflecting mirror (wave combiner) is introduced or not, at the point of intersection of the two partial waves, when the photon reached the wave combiner. The switching function of the cold neutron pulser could be used for that purpose. We have done the delayed choice experiment using the cold neutron spin interferometer of the Jamin-type arrangement which is installed at the JRR-3M cold neutron guide tube of Japan Atomic Energy Research Institute (JAERI) [20].

This paper contains also comments on some current attempts to measure the longitudinal coherence length $[21,22]$ by staggering the flight-times between the two coherent partial waves corresponding to two spin eigenstates.

\section{Low field magnetic mirror}

The magnetic neutron devices for cold neutron optics are required to satisfy the following three conditions: (1) they should be as small as possible to set compactly in a restricted space, (2) the stray fields from magnetic devices should be small enough not to give magnetic effects to nearby devices, (3) they should control the neutron spin states easily and precisely. Neutron refractive index $n_{ \pm}$ 
in a material is given by

$$
\begin{aligned}
& n_{ \pm}^{2}=1-\xi \\
& \xi=\left(V_{\mathrm{N}} \pm V_{\mathrm{m}}\right) / \mathrm{E} \\
& V_{\mathrm{N}}=\frac{2 \pi \hbar^{2} b_{\mathrm{N}} \rho}{m}=2.56 \times 10^{-18}\left[\mathrm{eV} \cdot \mathrm{cm}^{2}\right]\left(b_{\mathrm{N}} \rho\right)\left[\mathrm{cm}^{-2}\right] \\
& V_{\mathrm{m}}=\mu B=6.03 \times 10^{-12}[\mathrm{eV} / \text { gauss }] B \text { [gauss], }
\end{aligned}
$$

where $\mu$ is the magnetic moment of the neutron, $E$ - neutron energy in vacuum, $\rho$ - the number density of a mirror material, $b_{\mathrm{N}}-$ a coherent scattering length of the nuclei, $B-$ a saturation induction of a ferromagnetic material. Figure 1 shows the potential of the permalloy $(\mathrm{Fe}(55 \%) \mathrm{Ni}(45 \%))$.

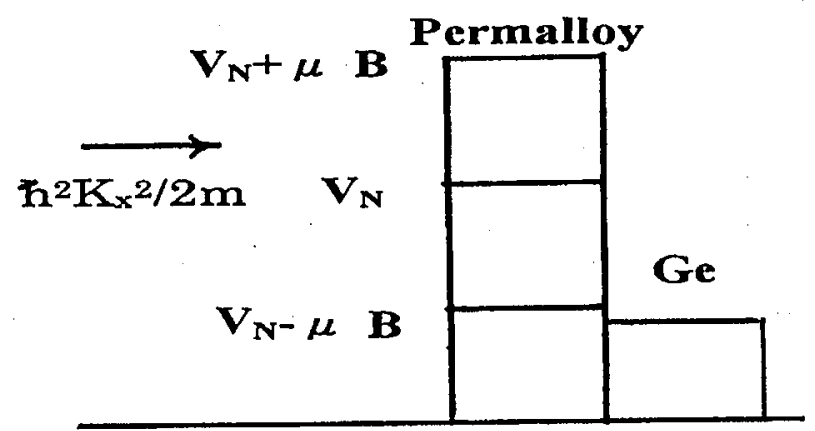

Fig. 1. Schematic one-dimensional potentials of permalloy $/ \mathrm{Ge}$ multilayer. $V_{\mathrm{N}}=220$, $93[\mathrm{neV}]$ for permalloy and germanium, respectively and $V_{\mathrm{N}}+\mu B=316[\mathrm{neV}]$, $V_{\mathrm{N}}-\mu B=123[\mathrm{neV}]$ for permalloy.

If the magnetic field $B$ is inhomogeneous, the force $F$ exerts on the neutron

$$
F= \pm \mu \nabla B(r) \text {. }
$$

This holds only in the case where the neutron's motion is so slow that magnetic mo-. ment maintains the same orientation with $B$ (adiabatic condition). The condition for this is that the time dependence of the field is much less than the precession frequency of magnetic moment of neutron,

$$
\frac{1}{\tau}=\frac{1}{B}\left|\frac{\partial B}{\partial t}\right| \ll \frac{2 \mu B}{\hbar}=\omega_{\mathrm{L}}
$$

If $\partial B / \partial t=0$, the Hamiltonian of neutron does not explicitly depend on time and thus the total energy of the neutron is conserved, whatever the spatial distribution of the field along the neutron trajectory may be. The change of the potential energy induced by the spatial variation of a static magnetic field brings about an equivalent change of the kinetic energy of the neutron while its total energy to remain constant.

Permalloy/Ge multilayer neutron polarizer (PGM) was developed. This magnetic mirror works under a very low magnetic field around zero gauss [23]. It is fabricated by vacuum evaporation of materials onto a Si wafer in a magnetic field 


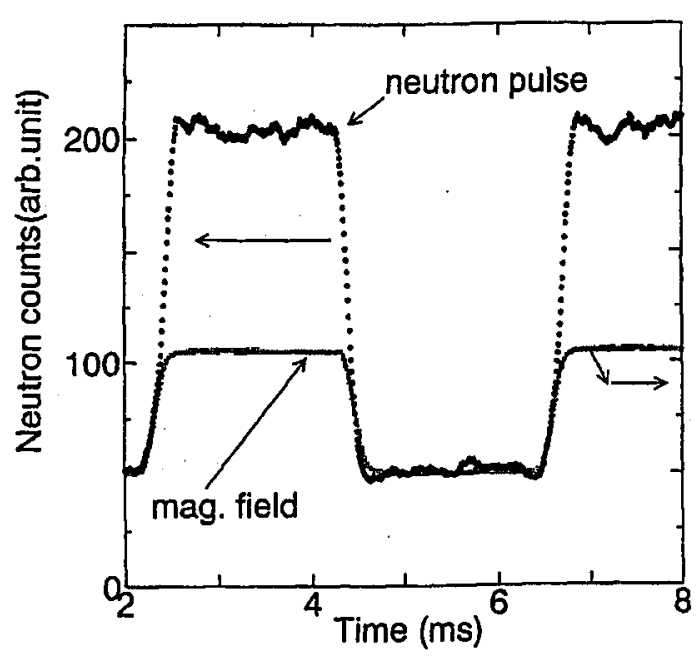

Fig. 2. Time spectra of the pulsed magnetic field and neutron pulses.

of about 100 gauss. The PGM consists of alternating parallel layers of permalloy and Ge. The refractive index of Ge for neutron is approximately equal to that of permalloy for neutron with spin aligned antiparallel to the magnetization of the permalloy layer. The bilayer consisting of permalloy layer and Ge layer functions as a single layer. The pulsed monochromatic beam can be generated if the magnetic induction orientation in a ferromagnetic layer can be changed by an external pulsing magnetic field as shown in Fig. 2. We call "a low field magnetic mirror placed in the pulsed magnetic field" a cold neutron pulser.

\section{Funahashi's interferometer of Jamin-type arrangement}

Figure 3 shows a schematic structure of Funahashi's interferometer which consists of a pair of composite mirrors. Neutron optical phenomena induced by a multilayer mirror are evaluated by the momentum component normal to the surface of the multilayer, since only the normal component undergoes effects of the potential changes at boundaries between layers. The phase difference (phase shift) $\phi$ between two coherent partial waves is given by

$$
\begin{aligned}
& \phi=\frac{4 \pi D n_{\perp}(\theta) \sin \theta}{\lambda} \\
& n_{\perp}(\theta)=\frac{k_{\perp}}{k_{0 \perp}}=\sqrt{1-\frac{b_{\mathrm{N}} \rho}{\pi}\left(\frac{\lambda}{\sin \theta}\right)^{2}},
\end{aligned}
$$

where $n_{\perp}(\theta)$ is the average refractive index of the effective gap layer for the normal component of the neutron momentum, $\theta-$ the neutron incident angle, $\lambda-$ the neutron wavelength, $\rho$ and $b_{\mathrm{N}}$ are the average atomic density and coherent scattering length of the effective gap layer, respectively. $k_{\perp}$ and $k_{0 \perp}$ are the wave numbers for the normal component of the neutron wave vector in the effective gap 


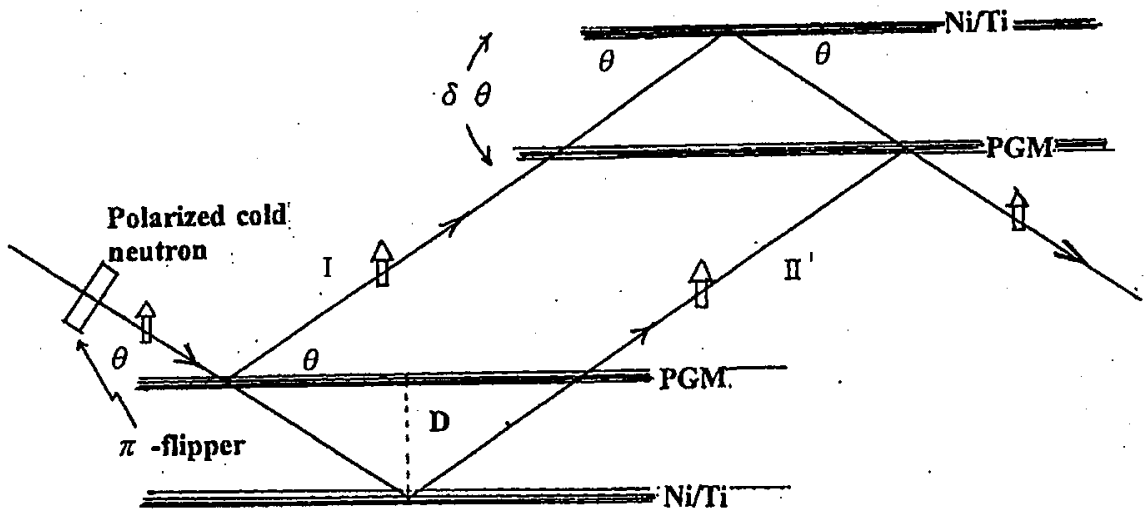

Fig. 3. Schematic view of the Jamin-type interferometer for a polarized neutron.

layer and air, respectively. The phase difference between the two partial waves induced through the first composite mirror (wave splitter) is compensated by the second one (wave combiner) because the two partial waves are superposed coherently upon each other at the second composite mirror. When we change the neutron incident angle $\theta$ onto a first or second composite mirror by $\Delta \theta$, the phase shift also changes. The change of the phase shift, $\Delta \phi$, is given by the equation, assuming $\theta \ll 1$ and $\Delta \theta \ll 1$

$$
\Delta \phi=\frac{4 \pi D}{\lambda}\left[n_{\perp}(\theta) \sin \theta-n_{\perp}(\theta+\Delta \theta) \sin (\theta+\Delta \theta)\right] \approx-\frac{4 \pi D \Delta \theta}{n_{\perp}(\theta) \lambda} .
$$

When $\Delta \theta$ is scanned from $-(\lambda / 2 D)(\lambda / \Delta \lambda)$ to $(\lambda / 2 D)(\lambda / \Delta \lambda)$, the interference fringes are observed with the periodicity of $\lambda / 2 D$ as shown in Fig. 4.

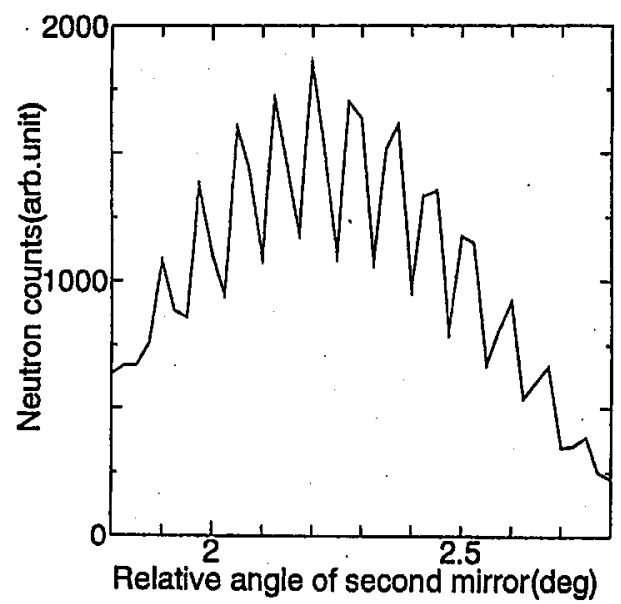

Fig. 4. Interference fringes observed by changing the angle of the second composite mirror relative to the first one in the Jamin-type interferometer. 


\section{Neutron spin-echo instrument as cold neutron spin-echo interferometer}

When the interaction energy between the neutron magnelic moment and the applied magnetic fields is smaller than the kinetic energy of neutron, the motion of neutron and spin are treated independently. So the Larmor precession of the spin $S=\hbar / 2$ is described by the classical equation of motion

$$
\begin{aligned}
& \frac{\mathrm{d} \boldsymbol{S}}{\mathrm{d} t}=\gamma \boldsymbol{S} \times \boldsymbol{B}, \\
& \gamma=2.916[\mathrm{kHz} / \mathrm{Oe}]=1.832 \times 10^{4}[\mathrm{rad} / \mathrm{s}] /[\mathrm{Oe}],
\end{aligned}
$$

where $\gamma$ is the gyromagnetic ratio equal to $\mu /|S|$. The solution of this equation in a time independent field $\boldsymbol{B}$ is well known as Larmor precession of which the Larmor precession angle $\phi$ is given by

$$
\phi=\phi_{0}+\omega_{L}(L / v),
$$

where $\omega_{\mathrm{L}}=\gamma B$ is the angular velocity.

In quantum mechanics the spin state of $S=1 / 2$ is described as the coherent superposition of two eigenstates, say $\uparrow$ and $\downarrow$. Let us consider a neutron polarized in the $x-y$ plane perpendicular to the magnetic field (quantization) direction $z$. The neutron state $\left|S_{x y}(\phi)\right\rangle$ and the expectation value of the $x$-component of the spin $\left\langle\mathrm{S}_{\mathrm{x}}(\phi)\right\rangle$ are expressed [11-13] as

$$
\begin{aligned}
& \left|S_{x y}(\phi)\right\rangle=\frac{1}{\sqrt{2}}\left[\left|\uparrow_{z}\right\rangle+\exp (\mathrm{i} \phi)\left|\downarrow_{z}\right\rangle\right]= \\
& \quad \exp \left(\frac{\mathrm{i} \phi}{2}\right)\left[\cos \left(\frac{\phi}{2}\right)\left|\uparrow_{x}\right\rangle-\mathrm{i} \sin \left(\frac{\phi}{2}\right)\left|\downarrow_{x}\right\rangle\right], \\
& \left\langle S_{x}(\phi)\right\rangle=\cos \phi,
\end{aligned}
$$

where $\phi$ is the phase difference between the two spin eigenstates, $\uparrow$ and $\downarrow$ states. The validity of this description was demonstrated experimentally by Summhammer et al. using a silicon interferometer $[14,15] . \phi$ expresses also the Larmor precession angle of the neutron spin. We call the spin precession expressed as the phase shift "quantum precession". Figure 5 shows the schematic structure of the spin-echo instrument. The neutron wave is split into two spin eigenstates by the polarizer and the first $\pi / 2$-flipper. The phase shift between two spin eigenstates changes through the two precession coils. Interference fringes are observed as an expectation value of the neutron spin with respect to $x$-axis. $\left\langle S_{x}(\phi)\right\rangle$ is measured by the second $\pi / 2$-flipper and an analyzer. Therefore, the spin-echo instrument is regarded as a sort of a neutron spin-echo interferometer. Our neutron spin-echo interferometer has the following features different from conventional spin-echo instrument: (1) the low field magnetic mirrors are used and the magnetic field applied to the system is. also low, (2) the rotational number of the neutron spin (Larmor precession number) is small, since the applied field is very low, and thus visibility is high, (3) these facts allow us easily to construct a neutron spin interferometer and to observe the various spin interferences between two spin eigenstates. Figure 6 shows the typical interference pattern of a cold neutron spin-echo instrument. 


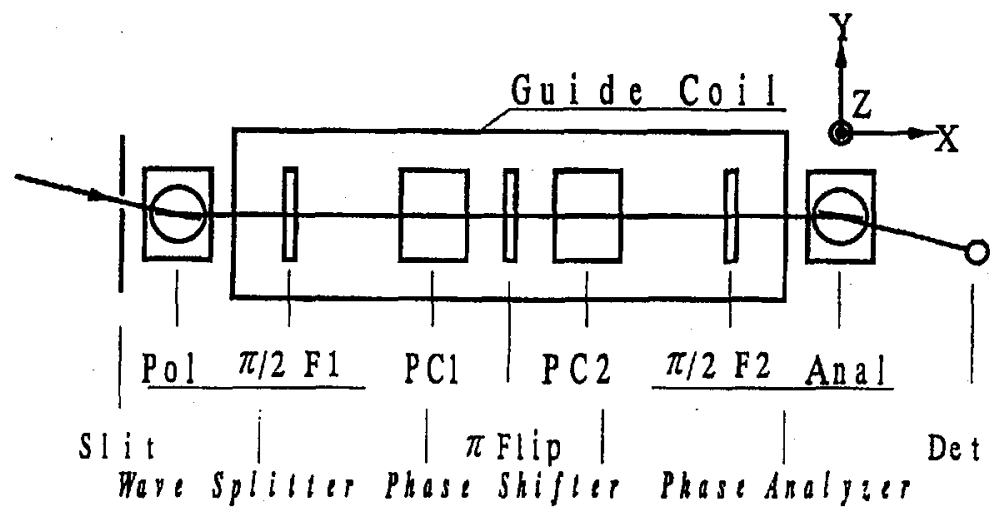

Fig. 5. Illustrative diagram of a spin-echo instrument as a cold neutron spin-echo interferometer.

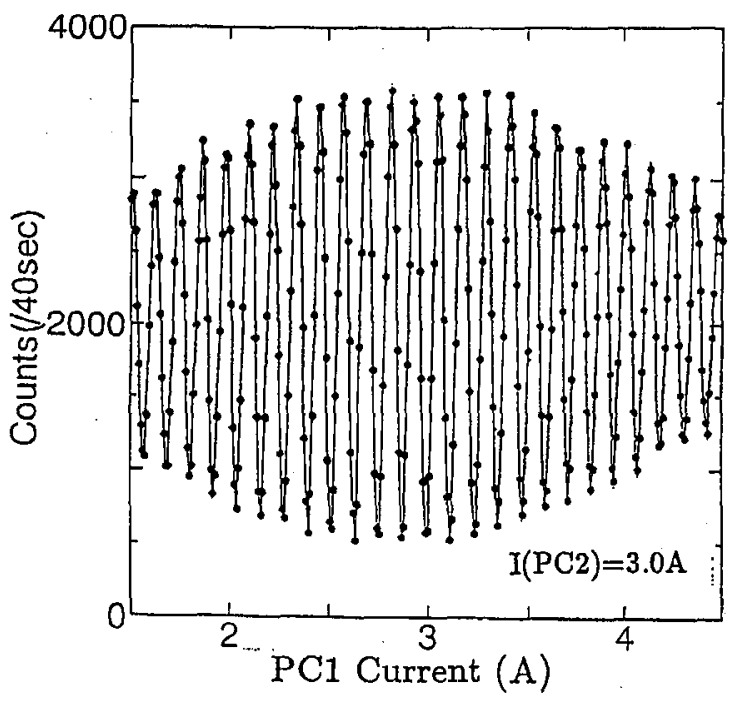

Fig. 6. Measured spin-echo profile as a function of PC1 coil current at a fixed current of $\mathrm{PC} 2$. One period of the profile corresponds to a change of $2 \pi$ in the Larmor precession in the magnetic field.

\section{Multilayer neutron spin splitter}

We consider a multilayer composite mirror consisting of a multilayer magnetic mirror with a high reflectivity $(\geq 90 \%)$ for the $+1 / 2$ spin component of the neutron and with a sufficiently low reflectivity for the $-1 / 2$ spin component, a gap layer and a multilayer non-magnetic mirror with a high reflectivity $(\geq 90 \%)$ for the neutron (the $-1 / 2$ spin component in this case). We call this a multilayer neutron spin splitter (MSS). When a neutron polarized in the $(x, y)$ plane is incident on a MSS, the $+1 / 2$ spin component of the neutron is reflected by the outer magnetic mirror while the $-1 / 2$ spin component is reflected by the inner non-magnetic mir- 
ror. An additional phase shift between the two spin eigenstates is produced by the effective gap thickness, $D$, which is taken to be equal to the thickness of the gap layer added to half the total thickness of the two multilayer mirrors [7].

The phase shift originates in the difference of path lengths between the two partial waves with different spin eigenstates as already given by Eq. (3.1). When the multilayer monochromators with a period $d$ are used as the two multilayer reflecting mirrors of MSS, Eq. (3.1) is reduced to Eq. (5.1) taking account of the Bragg condition for the monochromators, $\lambda=2 d \sin \theta$,

$$
\phi=\frac{2 \pi D n_{\perp}(\theta)}{d} .
$$

Equation (5.1) shows that only short distances are needed for the quantum precession because $d$ and $n_{\perp}$ are typically 100 to $200 \AA$ and 0.95 to 0.6 , respectively.

\section{Experimental demonstration of the equivalency of quantum precession and Larmor precession}

The arrangement for the performance tests of the multilayer spin splitters is shown in Fig. 7.

A multilayer spin splitter was set up on the goniometer in the magnetic field generated by PC2 coil so that the neutron incident angle can be scanned around the angle $\theta$ which satisfies the phase-echo condition. The spin-echo profiles were measured varying current of PC1 coil at a fixed current of PC2 coil.

We demonstrate that the change $\Delta \phi_{\mathrm{P}}$ of the quantum precession induced by an angular shift $\Delta \theta$ (given by Eq. (3.3)) can be compensated by an identical change $\Delta \phi_{\mathrm{L}}$ of the Larmor precession in the PC1 magnetic field.

However, we cannot obtain, usually, a spin-echo profile with an observable contrast around the conventional spin-echo condition, because the phase difference $\phi$ caused by a multilayer spin splitter entails a dispersion, $\delta \phi$, due to the wavelength distribution $\delta \lambda$ and the divergence angle, $\delta \theta$, of the incident neutrons. The dispersion of the phase difference, $\delta \phi$, is evaluated, neglecting the dependence of $\mathrm{n}_{\perp}(\theta)$ on $\theta$ and $\lambda$,

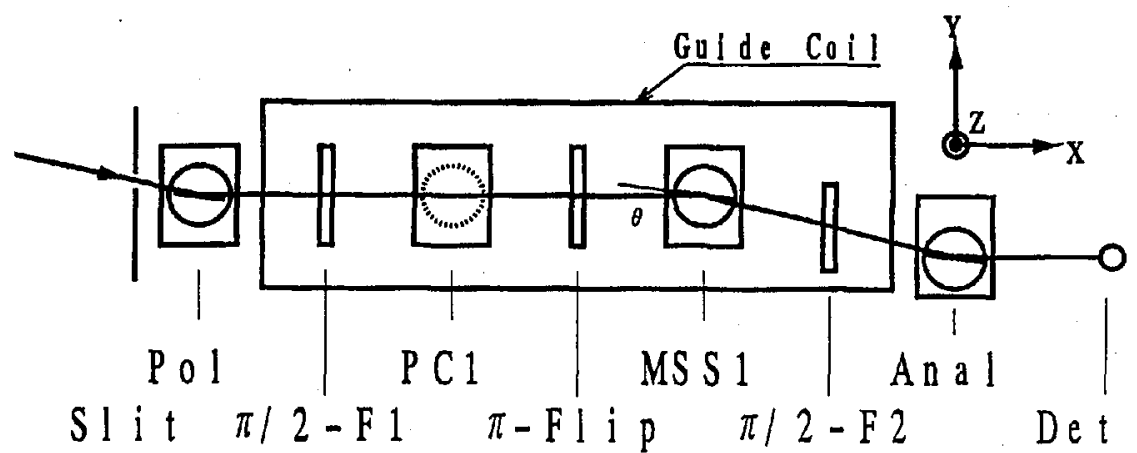

Fig. 7. Arrangement for a performance test of a multilayer spin splitter. The spin splitter is set up on the goniometer in the second precession field (PC2). The spin-echo profile is measured as a function of $\mathrm{PC} 1$ coil current under a fixed current of $\mathrm{PC} 2$. 


$$
\delta \phi=\frac{4 \pi D n_{\perp}(\theta) \sin \theta}{\lambda}[-(\delta \lambda / \lambda)+(\cot \theta) \delta \theta] .
$$

As $(D \sin \theta) / \lambda$ increases, the dispersion $\delta \phi$ becomes larger and larger depending on $\delta \lambda / \lambda$ and $(\cot \theta) \delta \theta$, although the wavelength dispersion dominates. A large dispersion would average out the contrast of the spin-echo profile.

The dispersion of the phase difference $\delta \phi_{\mathrm{P}}$, which originates from the wavelength distribution $\delta \lambda$ of the incident neutrons is compensated with an extra Larmor precession in the PC1 magnetic field and the contrast of the interference profiles is restored. This excess Larmor precession means the deviation from the normal spin-echo condition without a MSS due to the multilayer spin splitter.

Expressing the extra Larmor precession for a wavelength $\lambda$ by $\phi_{\mathrm{L}}$, the dispersion of the phase shift $\delta \phi_{\mathrm{P}}$ and that of the Larmor precession $\delta \phi_{\mathrm{L}}$ are given by Eq. (6.2) and Eq. (6.3), respectively, for a wavelength $\lambda+\delta \lambda$ deviating by $\delta \lambda$ from the center of the wavelength $\lambda$, to lowest order in $\delta \lambda / \lambda$

$$
\begin{aligned}
& \delta \phi_{\mathrm{P}}=4 \pi D n_{\perp}(\theta) \sin \theta\left(\frac{1}{\lambda}-\frac{1}{\lambda+\delta \lambda}\right) \approx \frac{4 \pi D n_{\perp}(\theta) \sin \theta}{\lambda}(\delta \lambda / \lambda), \\
& \delta \phi_{\mathrm{L}}=\frac{\phi_{\mathrm{L}}}{\lambda}[(\lambda+\delta \lambda)-\lambda]=\phi_{\mathrm{L}}(\delta \lambda / \lambda),
\end{aligned}
$$

where the sign of Eq. (6.3) is reversed owing to the $\pi$-flipper.

The restoration of the contrast of spin-echo profile depends on the cancellation of $\delta \phi_{\mathrm{P}}$ by $\delta \phi_{\mathrm{L}}$. The maximum contrast of the profile should be expected for the following reduction of the Larmor precession in PC1 magnetic field from the conventional spin-echo condition

$$
\phi_{\mathrm{L}}=-\frac{4 \pi D n_{\perp}(\theta) \sin \theta}{\lambda} .
$$

It should be noted that the dependences of the dispersions on $\delta \theta$ and on terms of higher order in $\delta \lambda / \lambda$ are neglected.

\section{Results of the performance tests of a multilayer spin splitter}

Spin-echo profiles were taken as a function of the current of PC1 coil in the case of which a multilayer spin splitter was mounted in the fixed magnetic field of PC2. We observed how the contrast of spin-echo profiles depends on the current of PC1 coil.

Figure 8 shows the spin-echo profiles as a function of $\mathrm{PC} 1$ current measured for the multilayer spin splitter $\mathrm{p} / \mathrm{G} 2 / \mathrm{N}$ which consists of two total reflection mirrors of permalloy- $45(\mathrm{P})$ and nickel $(\mathrm{N})$ with layer thicknesses of $700 \AA$, and a germanium (Ge) gap layer with a thickness of $2000 \AA$.

The multilayer spin splitter is set up in the fixed magnetic field of PC2. The abscissa indicates the PC1 coil current and the ordinate the neutron counts. The currents of PC2 coil were at values of $1.8 \mathrm{amp}$ and the neutron incident angle for $\mathrm{P} / \mathrm{G} 2 / \mathrm{N}$ is 1.17 degree, which is smaller than the critical total reflection angle of 1.20 degree.

When the current of the PC1 coil is equal to that of the PC2 coil, the conventional spin-echo condition is satisfied for the Larmor precession in the magnetic field. On the other hand, the multilayer spin splitter brings some large dispersion 


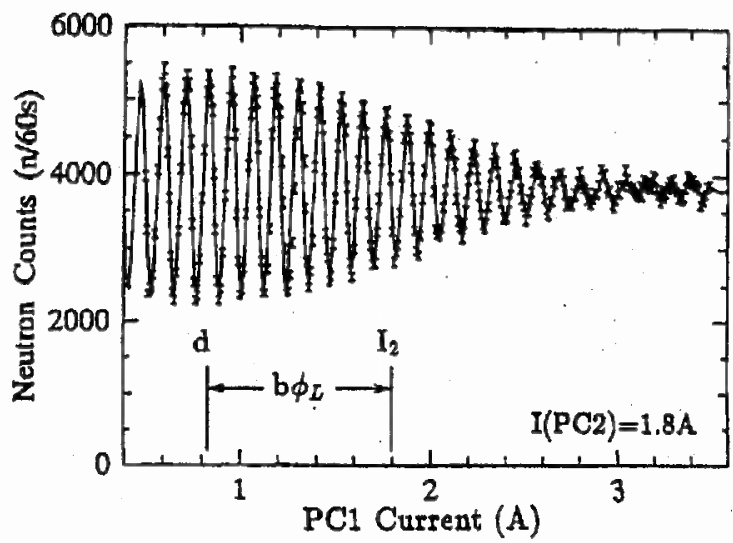

Fig. 8. Spin-echo profile for the multilayer spin splitter $(P / G 2 / N)$ as a function of $P C 1$ current under a fixed current of PC2 $(1.8 \mathrm{~A})$. The multilayer spin splitter is set up in the $\mathrm{PC} 2$. Restoration of the contrast of the spin-echo profile was observed in lower current region (around $0.8 \mathrm{~A}$ ) of $\mathrm{PC} 1$ comparing with the fixed current of PC2.

of the phase difference, as clescribed by Eq. (6.1). We, therefore, cannot observe any good contrast of the spin-echo profile due to the large dispersion. However, the contrast is restored when the current of the PC1 coil is reduced to less than that of the PC2 coil, as given by Eq. (6.4). The measured recovery of the contrast is related to the echo phenomenon between the phase difference and the Larmor precession, as previously described by Eqs. (6.2), (6.3) and (6.4).

This recovery of the contrast demonstrates that the quantum precession induced by the multilayer spin splitter is equivalent to the magnetic Larmor precession.

\section{A phase-spin-echo interferometer (cold neutron spin interferometer)}

Taking into account the equivalency of quantum precession by multilayer spin splitter and Larmor precession, a new type of cold neutron spin interferometer is considered. A pair of identical spin splitters, parallel to each other, are set up in a Jamin configuration, as shown in Fig. 9a, so the pair of multilayer spin splitters satisfies the phase echo condition [7] and the spin-echo condition [17] simultaneously. The simultaneous occurrence of the two echo phenomena is illustrated in Fig. 9b. The first multilayer spin splitter causes an additional phase difference between the two spin eigenstates given by Eq. (3.1). The $\pi$-flipper reverses the spin states of the two coherent partial waves. The reversed subbeams are reflected and superposed by the second spin splitter. As a result, the path length difference caused by the first multilayer spin splitter is compensated by the identical path length difference of the second one. We call this geometrical compensation a phase echo. The neutron polarization status after the reflection by the second spin splitter is restored completely by the phase-echo and the spin-echo phenomena. For the spin-echo phenomena we require a $\pi$-flipper. This restoration occurs for all incident neutrons independent of their angular and wavelength dispersion. So we call this system a phase-spin-echo interferometer or a cold neutron spin interferometer. 


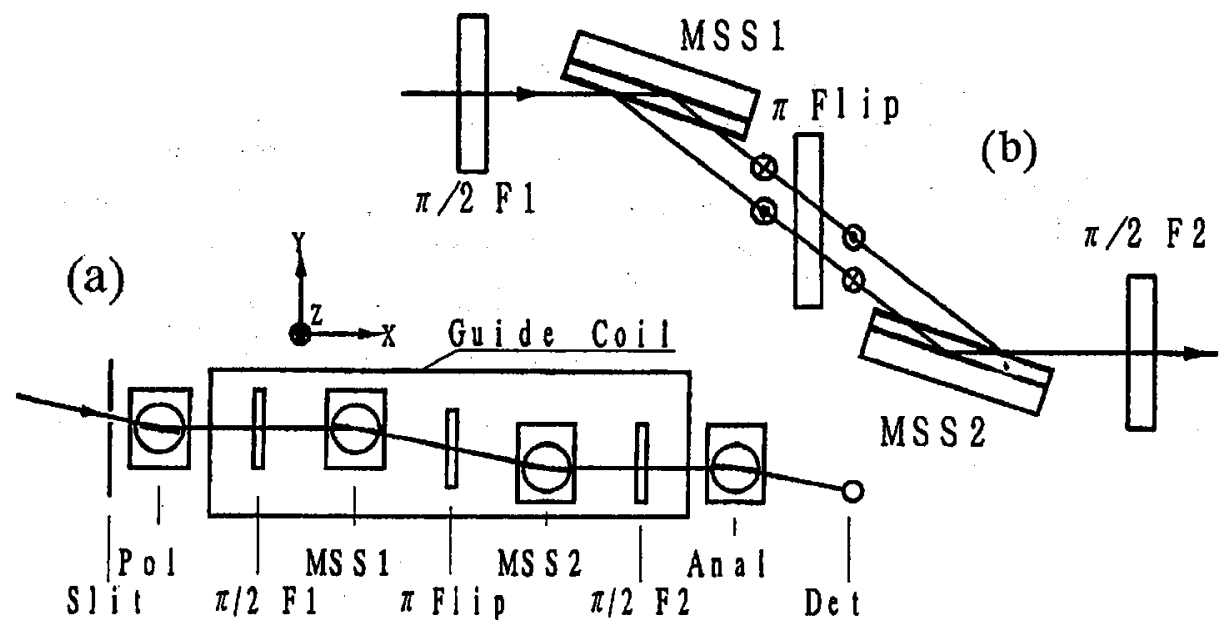

Fig. 9. (a) An arrangement of a phase-spin-echo interferometer consisting of a pair of identical multilayer spin splitters set up in the first and second precession coils, respectively. (b) Illustration of the simultaneous occurrence of phase- and spin-echo phenomena. The phase-echo requires a $\pi$-llipper.

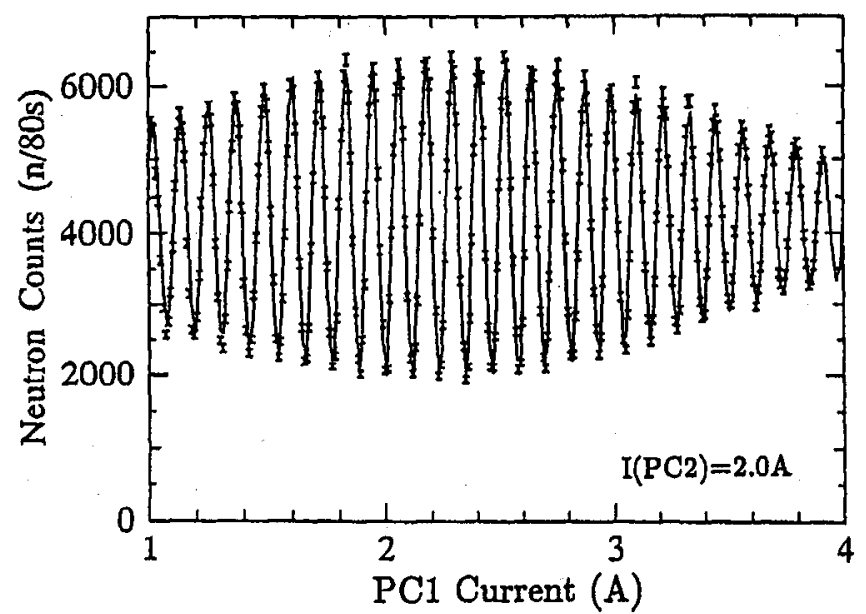

Fig. 10. Measured spin-echo profile for a phase-spin-echo interferometer. The contrast of the spin-echo profile is restored by the simultaneous occurrence of the phase- and spin-echo phenomena.

The measured spin-echo profile for the phase-spin-echo interferometer is shown in Fig. 10.

9. Delayed choice experiment using a cold neutron pulser

The composite neutron mirror consisting of a Ni/Ti multilayer ((Ni:85 $\AA /$ Ti:85 $\AA) \times 5$ (bilayers)), a Ge gap layer $(4000 \AA)$ and a permalloy $\left.\mathrm{Fe}_{55} \mathrm{Ni}_{45} \AA\right) / \mathrm{Ge}$ multilayer ((permalloy:85 $\AA /$ Ge:85 $\AA) \times 5$ (bilayers)) (PGM) was fabricated by 


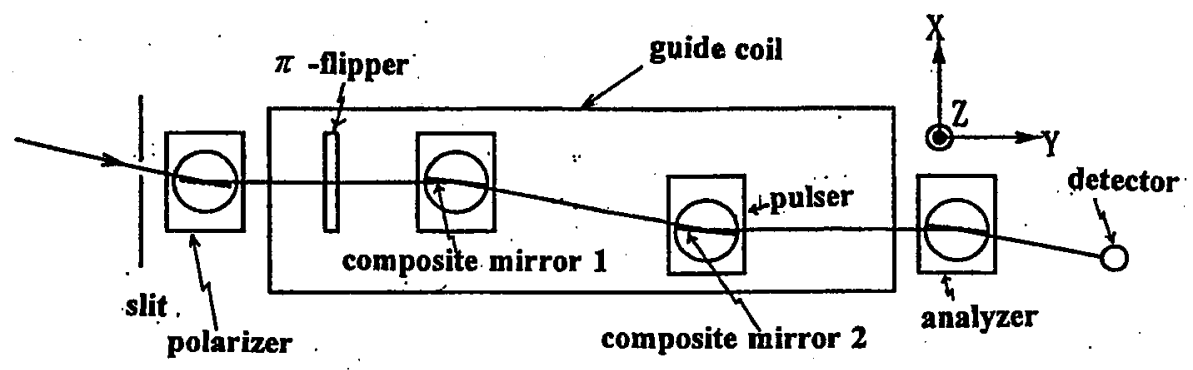

Fig. 11. Schematic arrangement of the delayed choice experiment. The pair of identical composite neutron mirrors composes the Jamin-type neutron spin interferometer.

evaporating in series on the single Si wafer in a magnetic field of 130 gauss. The calculated reflectivity of the PGM of 5 bilayers is about $10 \%$ [8]. The outmost PGM functions as a wave splitter for neutrons polarized in the direction parallel to the magnetic field (quantization axis) and the Ge gap layer plays the role of a phase shifter in the interferometer. The schematic experimental arrangement is shown in Fig. 11. The pair of identical composite neutron mirrors (wave splitters) was set at positions about $44 \mathrm{~cm}$ apart. Polarized $12.6 \AA$ neutrons with FWHM bandwidth of $3.5 \%$ are incident on the first wave splitter and divided into two coherent partial waves. The flipping ratio of the polarized neutrons was about 14/1. The phase difference between the two partial waves induced through the first wave splitter is compensated by the second one (wave combiner) because the two partial waves are superposed coherently upon each other at the wave combiner. When the second mirror rotates by a small angle $\delta \theta$ on a vertical axis parallel to the mirror surface, the phase difference changes, and interference fringes are observed by varying $\delta \theta$. This is the Jamin-type interference fringe as mentioned already.

In the case of the delayed choice experiment, the wave combiner is set in the pulsed magnetic field of 20 gauss to make a condition of whether the PGM of the wave combiner is introduced or not after a neutron passed through the wave splitter. The cold neutron pulser of PGM functions as a neutron optical switch.

Two delayed-choice modes are established: (1) the PGM of the wave combiner is not placed when the neutron reached the wave splitter, and it is introduced after the neutron passed through the wavesplitter, (2) the wave combiner was put when the neutron has reached the wave splitter, and it is removed after the neutron passed through the wave splitter. Whether the wave combiner is put or removed is decided after the neutron passed through the wave splitter. This is the meaning of "delaying choice". These modes were discriminated clearly by adjusting trigger pulse frequencies of the $\pi$-flipper and the current flowing into the pulsing coils, and the phase between them as shown in Fig. 12. The trigger pulse frequencies of the $\pi$-flipper and the current flowing into the pulsing coil of the second mirror are the same, that is $234 \mathrm{~Hz}$, of which half period corresponds to the neutron flight time from the $\pi$-flipper to the second mirror. In the first mode, the interference fringe was obtained and in the second mode the interference fringe disappeared as shown in Fig. 13. 


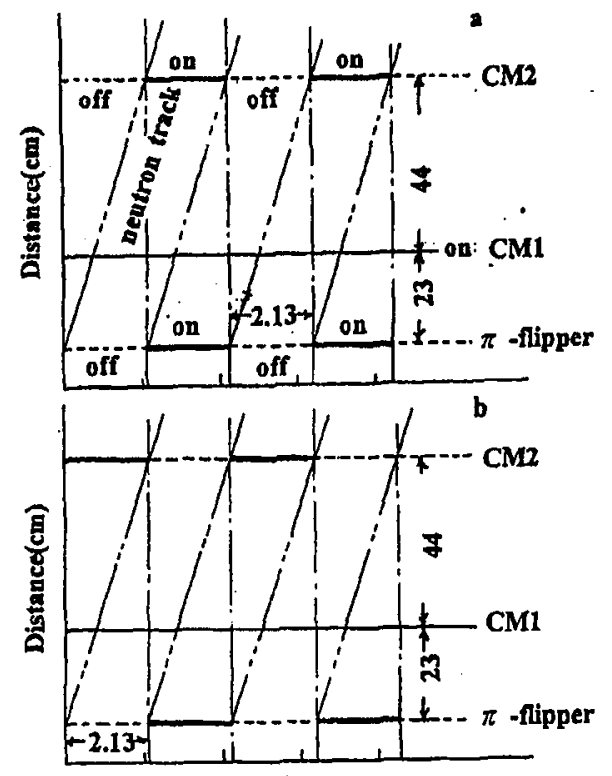

Time(ms)

Fig. 12. The time evolution in the delayed choice experiment. In the case (a), the PGM of second mirror (CM2) is not placed when the neutron reached the first mirror (CM1), and it is introduced after the neutron passed through CM1. In the case (b), the PGM of CM2 was placed when the neutron has reached CM1, and it is removed after the neutron passed through CM1.

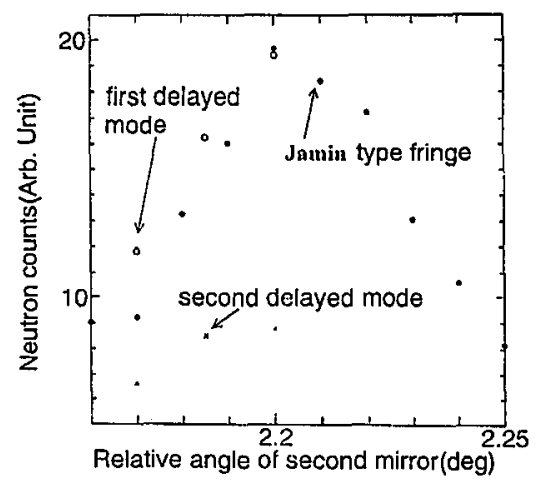

Fig. 13. The interference fringe observed by changing the relative angle between the first and the second mirrors. Interference fringe appeared in the first delayed mode, and disappeared in the second delayed mode.

These results show that whether the interference fringe is obtained or not depends only on the condition of whether the second wave splitter is introduced or not after the neutron passed through the first wave splitter. The delaying choice has no effect on the interference. From this result, we could deduce that the neutron wave propagates both ways with equal probability after being split by the first 
wave splitter and does not choose one of the two ways, as explained by the quantum mechanics.

\section{Attempts to measure the longitudinal coherence length using a time-resolved measuring method}

The direction of the magnetic induction of the PGM (quantization direction) is changed alternately applying the alternating high-frequency external field to the PGM (high-frequency cold neutron pulser), and thus the up and down spin components are reflected alternately from this pulsed magnetic mirror. By this method, we could generate the state consisting of two coherent spin eigenstates staggered in time. The longitudinal coherence length could be measured in combination of the high-frequency cold neutron pulser with the cold neutron spin-echo instrument as shown in Fig. 14. Figure 15 shows an experimental arrangement for measuring the effects of an overlap of the different spin eigenstates deviated transversally and in time to the longitudinal coherence length, that is, the effects of the transversal coherence length to the longitudinal one, by changing the thickness of the gap

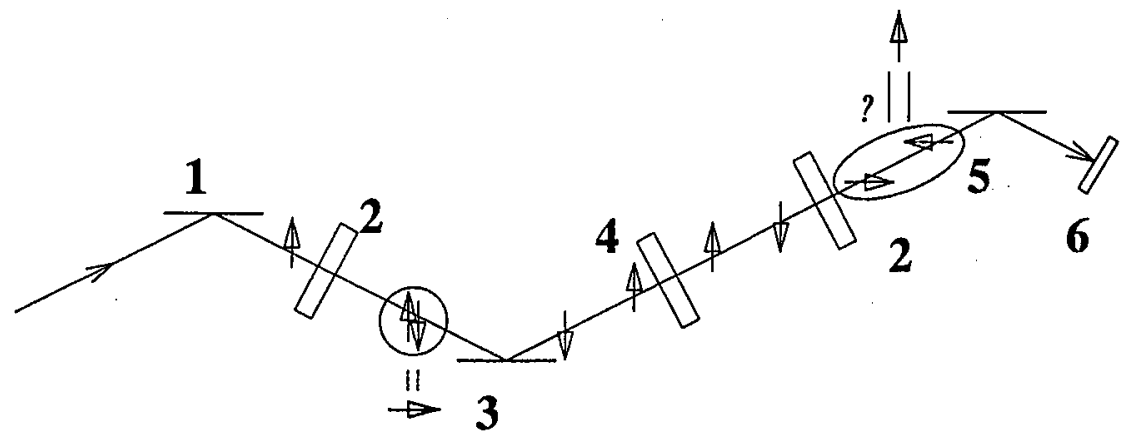

Fig. 14: Experimental arrangement for measuring the longitudinal coherence length. The elements of the instrument are: (1) polarizer, (2) $\pi / 2$-flipper, (3) high-frequency cold neutron pulser, (4) $\pi$-flipper, (5) analyzer, (6) scintillation counter.

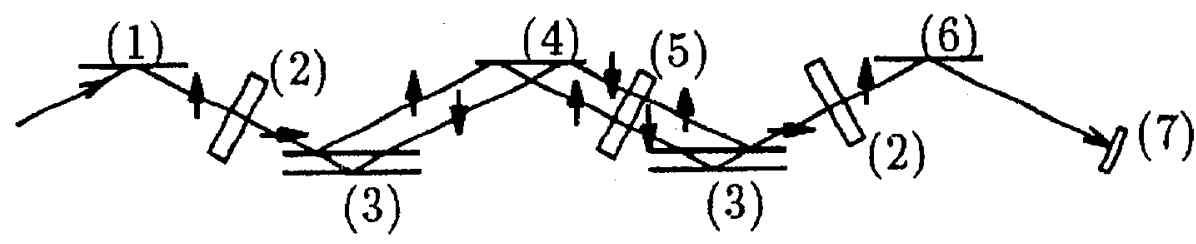

Fig. 15. Experimental arrangement for measuring the effects of an overlap of the different spin eigenstate deviated transversally and in time to the longitudinal coherence length. The elements of the instrument are: (1) polarizer, (2) $\pi / 2$-flipper, (3) multilayer spin splitter, (4) high-frequency cold neutron pulser, (5) $\pi$-flipper, (6) analyzer, (7) scintillation counter. 
layer. In this case, the phase difference between the two spin eigenstates, which is brought about by the geometrical path difference and their momentum difference due to Zeeman splitting, is compensated using a $\pi$-flipper. The time resolving technique is established by accurate synchronization and timing between the first $\pi / 2$-flipper and the high-frequency cold neutron pulser. From the reduction of contrast (visibility) of interference pattern, the longitudinal coherence length related to the time could be deduced. The high-frequency cold neutron pulser passing two coherent partial waves alternately in repetition time of $1 \mu \mathrm{s}$ is now developing.

\section{Acknowledgments}

I would like to thank Drs Toru Ebisawa, Seiji Tasaki, Masahiro Hino for daily stimulating discussions. This work was supported in part by the Inter-University Program for Common Use JAERI Facility and by the Common Use Program for KUR Facility, and financially by the Grant in Aid for Scientific Research of the Ministry of Education, Science and Culture in Japan (program numbers of 04244103 and 08404014).

\section{References}

[1] T. Ebisawa, H. Funahashi, S. Tasaki, Y. Otake, T. Kawai, M. Hino, N. Achiwa, T. Akiyoshi, J. Neutron Research 4, 157 (1996).

[2] T. Kawai, T. Ebisawa, S. Tasaki, Y. Eguchi, M. Hino, N. Achiwa, J. Neutron Research 5, 123 (1997).

[3] T. Ebisawa, S. Tasaki, T. Kawai, M. Hino, T. Akiyoshi, N. Achiwa, Y. Otake, H. Funahashi, J. Phys. Soc. Jpn. Suppl. A 65, 66 (1996).

[4] M. Hino, N. Achiwa, S. Tasaki, T. Ebisawa, T. Kawai, T. Akiyoshi, J. Phys. Soc. Jpn. Suppl. A 65, 281 (1996).

[5] T. Kawai, T. Ebisawa, S. Tasaki, T. Akiyoshi, M. Hino, N. Achiwa, Y. Otake, H. Funahashi, J. Phys. Soc. Jpn. Suppl. A 65, 230 (1990).

[6] T. Kawai, T. Ebisawa, S. Tasaki, M. Hino, D. Yamazaki, H. Tahata, T. Akiyoshi, Y. Matsumoto, N. Achiwa, Y. Otake, Physica B 241-243, 133 (1998).

[7] H. Funahashi, T. Ebisawa, T. Haseyama, M. Hino, A. Masaike, Y. Otake, T. Tabaru, S. Tasaki, Phys. Rev. A 54, 649 (1996).

[8] S. Tasaki, T. Kawai, T. Ebisawa, J. Appl. Phys. 78, 2398 (1995).

[9] T. Ebisawa, S. Tasaki, T. Kawai, T. Akiyoshi, M. Utsuro, Y. Otake, H. Funahashi, N. Achiwa, Nucl. Instrum. Methods A 344, 597 (1994).

[10] M. Hino, N. Achiwa, S. Tasaki, T. Ebisawa, T. Kawai, T. Akiyoshi, Physica $B$ 241-243, 1083 (1998).

[11] E.P. Wigner, Am. J. Phys. 31, 6 (1963).

[12] A. Zeilinger, Z. Phys. B 25, 97 (1976).

[13] A.Zeilinger, Neutron Space Interferometry, Eds. U. Bonse, H. Rauch, Clarendon Press, Oxford 1979, p. 241.

[14] J. Summhammer, G. Badurek, H. Rauch, U. Kischko, Phys. Lett. A 90, 110 (1982).

[15] J. Summhammer, G. Badurek, H. Rauch, U. Kischko, A. Zeilinger, Phys. Rev. A 27, 2523 (1983). 
[16] S. Tasaki, J. Appl. Phys. 71, 2375 (1992).

[17] F. Mezei, Z. Phys. 255, 146 (1972).

[18] T. Ebisawa, S. Tasaki, T. Kawai, M. Hino, N. Achiwa, Y. Otake, H. Funahashi, D. Yamazaki, T. Akiyoshi, Phys. Rev. A 57, 4720 (1998).

[19] J.A. Wheeler, Mathematical Foundations of Quantum Theory, Ed. A.R. Marlow, Academic Press, New York 1978, p. 9.

[20]. T. Kawai, T. Ebisawa, S. Tasaki, M. Hino, D. Yamazaki, T. Akiyoshi, Y. Matsumoto, N. Achiwa, Y. Otake, Nucl. Instrum. Methods A 410, 259 (1998).

[21] J. Felber, R. Gähler, R. Golub, Physica B 151, 135 (1988).

[22] H. Rauch, H. Wölwitsch, R. Clothier, H. Kaiser, S.A. Werner, Phys. Rev. A 46, 49 (1992).

[23] T. Kawai, Acta Phys. Pol. A 96, 19 (1999). 